\title{
PRESCRIPTION READING SYSTEM FOR VISUALLY IMPAIRED PEOPLE USING NLP
}

\author{
Neha Sahu, Akanksha Raut, Saloni Sonawane, Raza Shaikh \\ Department of Computer Engineering \\ Sandip Institute of Technology and Research Centre, \\ Nashik, Maharashtra, India
}

\begin{abstract}
A camera-based assistive text reading framework is proposed for visually impaired people to read the text labels from the Prescription in their day to day lives. In the proposed system, the camera acts as the main vision to capture the image of Prescription. To isolate the Prescription Image from complex backgrounds, we first propose a motion-based method to define region of interest (ROI) image. Then text characters are recognized by optical character recognition (OCR). The extracted texts are converted into audio output using the text to speech converter.
\end{abstract}

Keywords- Motion based method, Text Localization, Text Recognition, Optical Character Recognition

\section{INTRODUCTION}

Reading is essential in today's world. We see printed text everywhere in our surroundings. In our day to day lives we see newspapers, receipts, slips, menu cards, product packages, wrappers, medicines, reports, medical prescriptions etc. In order to help visually impaired people we have optical aids, video magnifiers, and screen readers which can help visually impaired people and the people with low vision. Braille is a system for reading and writing

through touch for visually impaired in which the letters of the alphabet are represented with raised dots. Equivalents for punctuation is also present in braille. The hands are moved from left to right over each line to feel the characters using braille. The visually impaired people can feel printed labels using Braille. There are few devices such as BrailleNote Touch Plus, Bluetooth Braille Displays etc. that can provide help to these people to carry out their work easily. There are recent developments in the field of digital cameras, computer vision and portable computers. These developing camerabased products combine various technology with some existing commercial products to make it feasible to assist the visually impaired individuals. The product label reading system is a system which helps the visually impaired to feel the text labels in their daily lives. An efficient and effective method has been proposed to define a region of interest to isolate the objects. Text localization and recognition are conducted to acquire the text information from the extracted ROI. The recognized texts are output to visually impaired people in the form of speech.

\section{MOTIVATION}

There exist a few systems that have some promises for their portable use, but they cannot handle product labeling. For example, portable barcode readers are designed that help visually impaired people to identify different products with ease. Product databases can enable the visually impaired users to access the information about the various products. However a big limitation is that it is very difficult for visually impaired people to find the position of the bar code and to correctly point the bar code reader at the bar code scanner. Some reading systems like pen scanners, mobile readers might be deployed in these similar situations. OCR software is integrated with these systems to offer scanning and recognition function of text; also some systems may have integrated voice results. However, these kinds of systems perform best with the text images with simple backgrounds, standard fonts, a range of font size and well-organized characters rather than the hand held product packages with various patterns. Most of the OCR software cannot directly handle the images with complex backgrounds. A number of reading assistants systems have been designed specifically for the visually impaired people, but still no existing reading assistant can read text from the complex backgrounds found on many everyday commercial products.

\section{PROPOSED ARCHITECTURAL DESIGN}

Module 1: Scan Prescription and Pre-processing 


\section{International Journal of Engineering Applied Sciences and Technology, 2020 Vol. 4, Issue 12, ISSN No. 2455-2143, Pages 265-268 \\ Published Online April 2020 in IJEAST (http://www.ijeast.com)}

The user scans the prescription in the application installed in the mobile with the help of a camera. After Scanning the image, the application will preprocess the image in the database. Pre-processing is the lowest level of abstraction in the image. The aim of pre-processing is to improve the image data that suppresses unwanted distortions or enhances some image features important for further processing. Noise filtering process is then applied to eliminate the noise present in the image.

Module 2: Edge Detection, Region of Interest and Segmentation.

Edge detection is an image processing technique used for finding the boundaries of objects within images. It works by detecting discontinuities in brightness. Edge detection is used for segmentation of the image and extraction of the data in image processing. A region of interest (ROI) is a portion of an image that needs to be filtered. ROI is defined by creating a binary mask, which is a binary image that is the same size as the image to be processed with pixels. The ROI set to 1 where the characters are present in the particular cell of the matrix and all other pixels are set to 0 .

To simplify and change the representation of an image into something that is more meaningful and easier to analyze is the goal of segmentation. Image segmentation is used to locate objects and boundaries (lines, curves, etc.) in images. More precisely, image segmentation is the process of assigning a label to every pixel in an image such that pixels with the same label share certain characteristics.

\section{Module 3 : OCR using NLP}

After segmenting the image the OCR (Optical Character Recognition) Algorithm is applied using NLP (Natural Language Processor)

\section{STEPS OF OCR Algorithm:-}

\section{Image Acquisition:}

The first step is to acquire images of paper documents with the help of scanners. This way, an original image can be captured and stored. Most of the paper documents are black and white, and an OCR scanner should be able to threshold images. In other words, it should replace each pixel in an image with a black or a white pixel. It is a method of image segmentation.

\section{Preprocessing:}

The goal of preprocessing is to make raw data usable by computers. The noise level on an image should be optimized and areas outside the text removed. Preprocessing is especially vital for recognizing handwritten documents that are more sensitive to noise. Preprocessing allows obtaining a clean character image to yield better results of image recognition.

\section{Segmentation:}

The process of segmentation is aimed at grouping characters into meaningful chunks. There can be predefined classes for characters. So, images can be scanned for patterns that match the classes.

\section{Feature Extraction:}

This step means splitting the input data into a set of features, that is, to find essential characteristics that make one or another pattern recognizable. As a result, each character gets classified in a particular class.

\section{Post-Processing:}

This stage is the process of refinement as an OCR model can require some corrections. However, it isn't possible to achieve 100 percent recognition accuracy. The identification of characters heavily depend on the context. The verification of the output requires a human-in-the-loop approach.

Module 4 : Meaningful word recognition

In module 4 after applying the OCR Algorithm on image all the meaningful words are extracted from it. These words are formed into sentences for speech conversion to the users.

\section{Module 5: Text to Speech}

The image is converted into speech output to the user in the form of audio. Refer fig .1 
International Journal of Engineering Applied Sciences and Technology, 2020

Vol. 4, Issue 12, ISSN No. 2455-2143, Pages 265-268

Published Online April 2020 in IJEAST (http://www.ijeast.com)

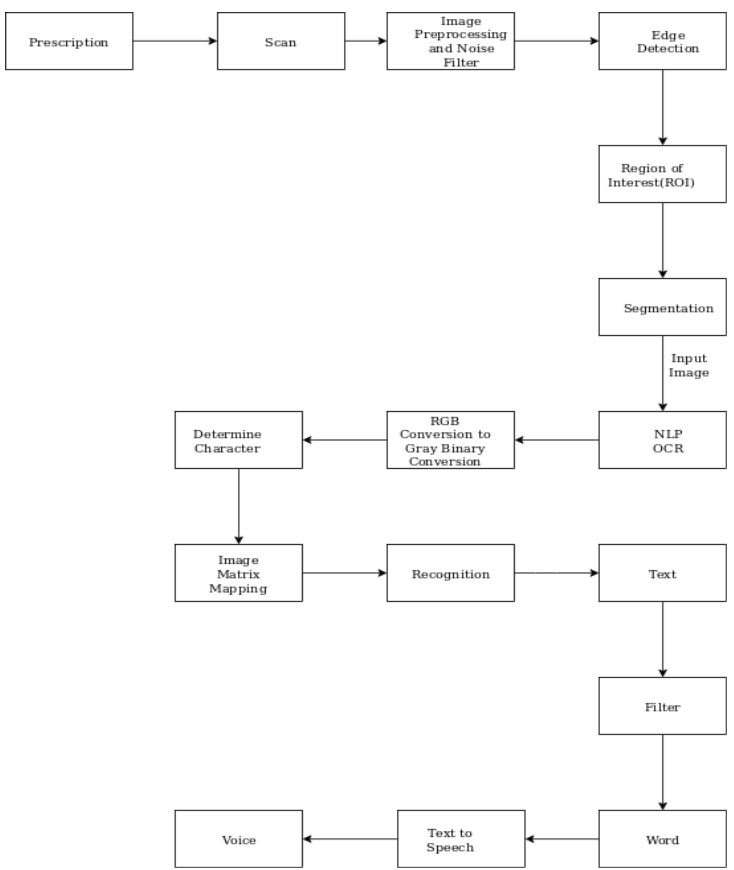

Fig.1 flow chart.

\section{PROPOSED ALGORITHM}

STEP 1: Capture an image by using a camera.

STEP 2 : In captured image, System detects Region of interest (ROI).

STEP 3 : Extracts Text Region.

STEP 4 : Extracted text region undergoes text binarization and recognition.

STEP 5 : Text recognition is performed by OCR.

STEP 6 : Displaying label in the form of text.

STEP 7 :Text is converted to speech .

\section{RESULTS}

Once the prescription is scanned the medicine name is copied in the medicine name field in the application. Thereafter users can set the days for medicine intake as prescribed by the doctor. The reminder for medicine intake time is set according to the doctors prescription Refer Fig 2.1

The phone will beep a reminder through the application and display the time set by the user along with the medicine name. The medicine name given as text input is then converted into audio form and the application will call out the name of the medicine. Refer fig. 2.2.

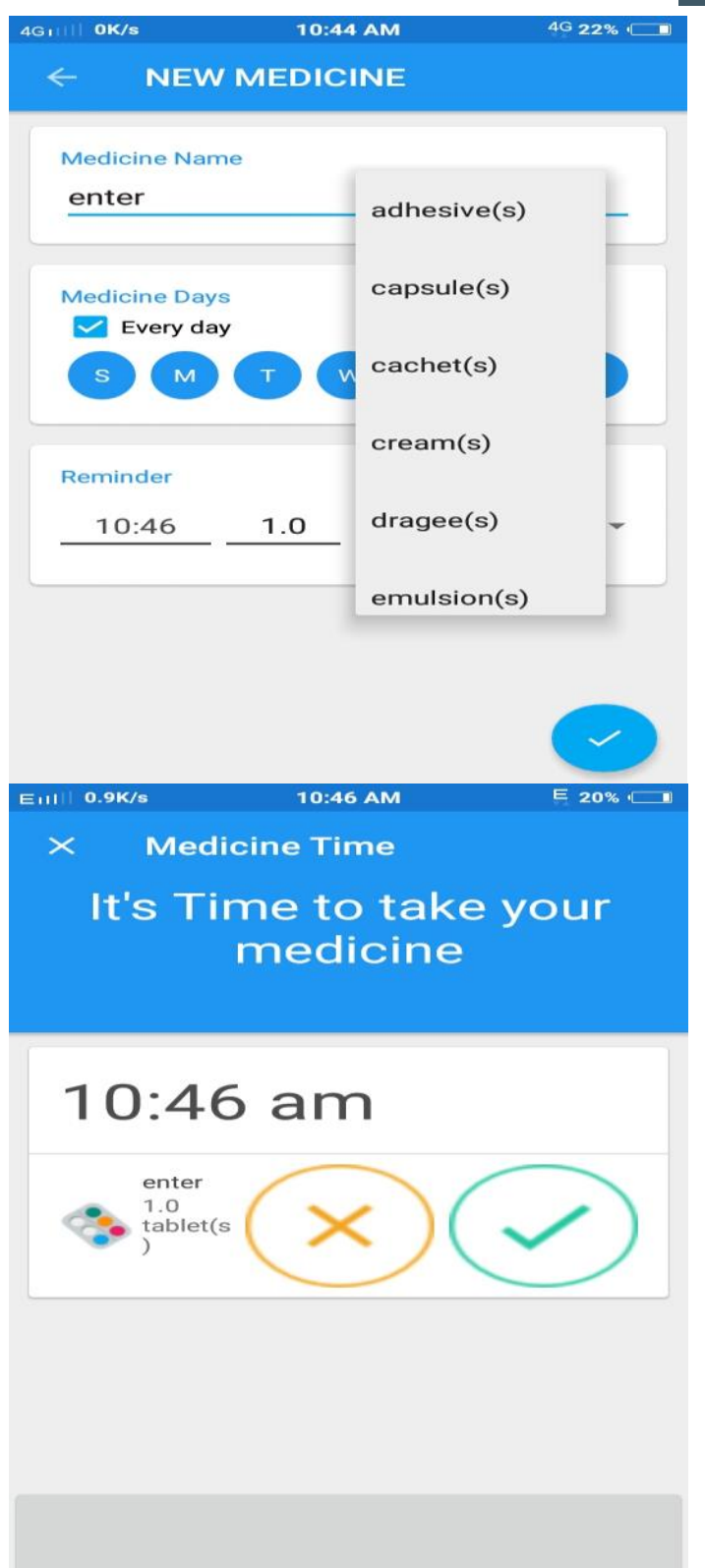

Fig 2.1(new medicine added) Fig 2.2(Alarm for taking doses)

\section{CONCLUSION}

1. The existing literature has been studied for current problems of interest.

2. To overcome the challenges in existing system or technology, a new Prescription Reading for Visually Impaired system is proposed using NLP

3. The Proposed System will use OCR (optical Character Recognition).

4. The feasibility of Audio Output by OCR is verified. Moreover, the method can effectively solve the problem of Reading Prescription caused by the visually impaired people and has a great effect on them. 


\section{International Journal of Engineering Applied Sciences and Technology, 2020 Vol. 4, Issue 12, ISSN No. 2455-2143, Pages 265-268 \\ Published Online April 2020 in IJEAST (http://www.ijeast.com)}

\section{REFERENCE}

[1] Rahulkumar Chhatwani, Arvind Langhe (2017): "Product Label Reading System for visually challenged people" ,International Research Journal of Engineering and Technology (IRJET), Volume: 04.

[2] Sapate V. D. (2016):"A Survey: Text Extractionfrom Images and Video" ,International Journal of Advanced Research in Computer and Communication Engineering, Volume: 05.

[3] N.Itunuoluwa sewon, Jelili Oyelade Olufunke Oladipupo PMB 1023, Ota, "Design and Implementation of Text To Speech Conversion for Visually Impaired People".

[4] ChawSuThuThu T. Z.: "Implementation of Text to Speech Conversion".

[5] Ayushi Trivedi P. S. S. S., Navya Pant and S.A. C. A. Pant:"Speech to text and text to speech recognition systems-A Review".

[6] I.-O. N. A. and A. A. A. :"A Voice-based Mobile Prescription Application for Healthcare Services (VBMOPA)".

[7] Randhir Jagannath Patil D.S.: "Voice Based Medicine Prescription".

[8] Rajkumar B. N., Anand M.G :'Portable CameraBased Product Label Reading For Blind People".
[9] Chucai Yi I. Y. T. S. M. I. A. A. , Student Member (2016) : "Portable Camera-based Assistive Text and Product Label Reading from Hand-held Objects for Blind Persons" ,International Journal of Advanced Research in Computer and Communication Engineering, Volume: 05.

[10]Jayashree A. P. Dr.D., Afritha Farhath.K (2016): "Voice Based Application As Medicine Spotter For Visually Impared", Second International Conference on Science Technology Engineering and Management (ICONSTEM).

[11] Karen Duarte J. S. S. P. F., Jos'eCec'1lio (2014): "Information and Assisted Navigation System for Blind People",8th International Conference on Sensing Technology.

[12] Rajkumar N , Anand M.G ,Barathiraja N(2014): "Portable Camera-Based Product Label Reading For Blind People", International Journal of Engineering Trends and Technology (IJETT) Volume 10 Number 11

[13] Ashwini V. Mhaske, Mahesh S. Sadavarte(2016):'Portable Camera Based Assistive Text Reading from Hand Held Objects for Blind Person", International Journal of Advanced Research in Computer and Communication Engineering,Volume: 05. 